\title{
LEXICALIZACIÓN DE SECUENCIAS NOMBRE DEVERBAL-ADJETIVO RELACIONAL
}

\author{
Laura Kornfeld, Gabriela Resnik
}

\begin{abstract}
RESUMO: The present article focuses on the study of deverbal noun-relational adjective sequences such as contaminación hídrica or gasto ambiental.. First, we characterise the properties of the relational adjectives and the deverbal nouns that form the sequences and we distinguish two types of sequences, according to the distinction of Bosque \& Picallo (1996) between thematic and classificatory relational adjectives. From this classification, we revise the phenomenon of reduction (cf. Adelstein \& Kuguel, 1998). Finally, we propose possible terminographic applications of the above analysis.
\end{abstract}

PALAVRAS-CHAVE: lexicalização, sintagmas, derivação, eventividade, aplicações para o MERCOSUL.

\section{Introducción}

En un trabajo anterior (KORNFELD \& RESNIK, 1998), hemos analizado las secuencias de la forma nombre-preposición de-nombre que tienen un primer nombre deverbal, a partir de un corpus conformado por las entradas de un glosario que registra la terminología del medio ambiente ${ }^{1}$. El análisis de estas secuencias permitió determinar que se trata de construcciones que no presentan ningún grado de lexicalización formal, es decir, que son combinaciones sintácticas frecuentes (colocaciones) y no sintagmas lexicalizados. De acuerdo con la propuesta de Di Sciullo \& Williams (1987), en el trabajo mencionado concluimos que las secuencias del tipo contaminación del agua son objetos sintácticos, generados por reglas sintácticas, que aparecen en posición de frase y siempre son analizados internamente por la sintaxis. Por el contrario, los sintagmas lexicalizados del tipo calidad de vida constituyen instancias de palabras sintácticas, es decir, de secuencias generadas en la sintaxis, pero que, una vez constituidas, no son analizadas internamente por el componente sintáctico (i.e. son sintácticamente opacas), y funcionan como un átomo sintáctico, esto es, se insertan en bloque en posición nuclear. En Kornfeld \& Resnik 1999, los distintos grados de lexicalización formal de las secuencias nombre-preposición de-nombre se establecen en función de la posibilidad de realizar determinadas operaciones sintácticas (inserción del determinante, correferencia con un elemento externo, imposibilidad de agregar modificadores para alguno de los dos nombres).

En el presente trabajo, nos proponemos abordar secuencias de la forma nombre-adjetivo que podrían emparentarse con las que caracterizamos anteriormente como objetos sintácticos. Se trata de las secuencias nombre deverbal-adjetivo relacional, como contaminación ambiental, vinculado con contaminación del ambiente.. Por un lado, caracterizaremos las propiedades tanto de los adjetivos relacionales como de los nombres deverbales que componen las secuencias. A partir de esta caracterización, distinguiremos dos tipos de secuencias, en función de la distinción de Bosque \& Picallo (1996) entre adjetivos relacionales temáticos y clasificatorios. Por otro lado, veremos cómo esta clasificación permite revisar desde otra perspectiva el fenómeno de la reducción (cfe. ADELSTEIN \&

\footnotetext{
Laura Kornfeld e Gabriela Resnik são professoras da Universidade de Buenos Aires

1 Alonso, H., N. Bricchetto de Lara y R. Palota (coord.) (1995) Glosario Ambiental. Buenos Aires: Secretaría de Recursos Naturales y Ambiente Humano de la Nación (Sistema de consulta de Glosario Ambiental Versión 0).
} 
KUGUEL, 1998; ADELSTEIN, 1998; KUGUEL, 1998; ADELSTEIN, KUGUEL et al., 1999). Por último, propondremos posibles aplicaciones terminográficas del análisis presentado.

\section{Caracterización de las secuencias nombre deverbal-adjetivo relacional}

En función de las características morfológicas del adjetivo, podemos distinguir tres clases de secuencias nombre-adjetivo: con adjetivos no derivados (marea roja), con adjetivos deverbales (especie contaminante) y con adjetivos denominales (planificación ambiental). Dentro de esta última clase, las secuencias más frecuentes son las que contienen un adjetivo relacional (BOSQUE, 1990). (En el anexo incluimos los sintagmas de la forma N+A relevados del Glosario Ambiental, clasificados de acuerdo con las características morfológicas del adjetivo.)

Como se ha mencionado previamente, el tipo de secuencia que nos ocupará en este trabajo es la que presenta un núcleo nominal derivado de verbo que toma como complemento un adjetivo relacional. De acuerdo con la distinción establecida por Bosque\&Picallo 1996, existen dos clases de adjetivos relacionales: los temáticos y los clasificatorios. En el primer caso, el adjetivo satura un rol temático requerido por el núcleo nominal deverbal (pesca ballenera), mientras que en el segundo caso el adjetivo no absorbe un rol temático sino que introduce un dominio en relación con el cual se clasifica la entidad denotada por el núcleo nominal (análisis sintáctico). En la terminología del medio ambiente, dentro del grupo en el que el adjetivo temático hace referencia a la entidad física involucrada como paciente o agente, se ubican contaminación hídrica, control ambiental, cambio climático, daño ambiental, deterioro ambiental, emanación tóxica, erosión hídrica, erosión eólica, ordenamiento territorial, protección ambiental, vigilancia ambiental y presión demográfica, entre otros. En este caso, el adjetivo no denota una propiedad sino que realiza un argumento, de la misma manera que el nombre del que deriva. En contaminación hídrica, el adjetivo satura el rol temático "tema" que el núcleo nominal hereda de la grilla del verbo contaminar.

En el caso de los adjetivos clasificatorios, en cambio, el adjetivo se opone a otros adjetivos relacionales en una clasificación que incluye distintos dominios (por ejemplo, gasto ambiental por oposición a gasto educativo o gasto social). En gasto ambiental, el ambiente no es lo gastado ni tampoco es la entidad que gasta, sino que funciona como un adjunto que especifica respecto de qué debe entenderse el nombre gasto. Son ejemplos de este grupo de sintagmas, además de gasto ambiental, costo ambiental, costos sociales, enfoque ambiental, interpretación ambiental y difusión ambiental.

Las paráfrasis posibles de cada tipo de sintagma demuestran la relación temática en un caso y clasificatoria en el otro:

(1) La contaminación hídrica se produjo debido a los efluvios cloacales.

La contaminación de las aguas se produjo debido a los efluvios cloacales..

Los efluvios cloacales contaminaron las aguas.

Contaminación de las aguas resulta, entonces, un sinónimo de contaminación hídrica (de hecho, ambas secuencias figuran en el Glosario Ambiental). En el último ejemplo de (1), por otra parte, advertimos que es posible concebir una construcción verbal de sentido equivalente.

En cambio, no es aceptable una paráfrasis en la que ambiental se interprete como "tema" de gasto:

(2) Es necesario incrementar el gasto ambiental.

* Es necesario incrementar el gasto del ambiente.

La paráfrasis aceptable en este caso pone de manifiesto la idea de 'dominio' en el que se aplica gasto:

(3) Es necesario incrementar el gasto relativo al ambiente.

Cabe observar que gasto relativo al ambiente no es sino una definición mínima de gasto ambiental, y que constituye la única designación alternativa del concepto 'gasto ambiental' .

Asimismo, advertimos que los sintagmas cuyo adjetivo es clasificatorio admiten un complemento agentivo encabezado por $d e$, lo que no es posible en el caso de los sintagmas con un adjetivo temático:

(4) gasto ambiental del gobierno / difusión ambiental de la secretaría

*contaminación hídrica de la industria /*degradación ambiental de los desechos

Esta diferencia se explica por el hecho de que, en general, la realización del argumento interno heredado por el nombre deverbal (por medio de un sintagma adjetivo o de un sintagma preposicional) bloquea la realización de un sintagma de interpretación agentiva encabezado por la preposición de. El mismo fenómeno se produce en el léxico común: ?La narración de los hechos de Juan o ?La extracción dental del cirujano.

Vemos, entonces, que un mismo adjetivo relacional (ambiental, por ejemplo) puede funcionar de 
distinto modo de acuerdo con los requerimientos léxicos del núcleo nominal. Observamos que, en general, en los casos en que existen requerimientos temáticos, el sintagma mantiene propiedades eventivas, mientras que en los casos en los que no hay tales requerimientos el núcleo suele tener una interpretación resultativa.

Cabe señalar que un mismo deverbal puede funcionar como eventivo y como resultativo, como puede verse en los siguientes ejemplos:

(5) La organización del festival llevó tres meses.

(6) El evento contó con la participación de una organización no gubernamental.

Mientras que (5) admite paráfrasis verbal, (6) no:

(7) El festival se empezó a organizar en septiembre.

En (5) organización se interpreta como un proceso que puede especificarse temporal o aspectualmente. En (6), en cambio, organización se interpreta como la entidad resultante del proceso de organizarse.

La ausencia de eventividad se manifiesta además en el hecho de que el deverbal organización, en (6), puede pluralizarse.

(8) *Las organizaciones de los festivales llevaron tres meses.

(9) El evento contó con la participación de varias organizaciones no gubernamentales.

Así, en el caso de los nombres con -ción (construcción, redacción, descripción) el cambio en las propiedades semánticas del nombre (designación de una entidad) tiene como correlato un cambio en las propiedades morfológicas (pluralización), que representa el grado máximo de pérdida de eventividad en el deverbal ${ }^{2}$.

En los casos de gasto o difusión, si bien los deverbales no pierden las propiedades eventivas al punto de pasar a designar una entidad, se observa que, en contraste con contaminación o control, tienen una interpretación resultativa. Una prueba de la dificultad para asignar a estos nombres una interpretación eventiva es su incompatibilidad para funcionar como argumento interno de verbos como producirse o efectuarse como puede verse en el contraste entre (10) y (11):

(10) A causa del vertido de desechos tóxicos se produjo una contaminación hídrica.

El accidente fue el resultado de que no se hubiera efectuado un control ambiental.

(11) ?? Este año se produjo un gasto ambiental.

??La Secretaría de Recursos Naturales efectuó una difusión ambiental.

Los verbos producirse y efectuarse requieren que su argumento interno tenga rasgos eventivos; esto es, sólo un evento (o algo que se concibe como tal) puede producirse o efectuarse.. Observamos que, mientras contaminación hídrica y control ambiental satisfacen este requerimiento, gasto ambiental y difusión ambiental, que han perdido propiedades eventivas, producen la anomalía semántica de la oración.

Las diferencias presentadas hasta aquí muestran que las propiedades eventivas son relevantes para la determinación de las posibilidades de lexicalización de las secuencias compuestas por un nombre deverbal y un adjetivo relacional. En efecto, en las secuencias nominales, la ausencia y la presencia de rasgos formales y semánticos típicamente verbales están asociadas cada una con los extremos de una escala cuyos polos son +/- lexicalización. Es decir, mientras que la mayor lexicalización está ligada con los rasgos formales y semánticos típicos de los nombres (posibilidad de pluralización, designación de una entidad), la mayor independencia entre los componentes de la secuencia se encuentra ligada con los rasgos formales y semánticos propios de los verbos (imposibilidad de pluralización, eventividad). Las diversas paráfrasis de contaminación hídrica vistas en (1) manifiestan el hecho de que un evento puede ser expresado mediante múltiples combinaciones sintácticas que remiten a la misma estructura conceptual. En cambio, las posibilidades de parafrasear gasto ambiental se limitan a la definición mínima del sintagma (cfr. ejemplos (2) y (3)), análogamente a lo que sucede con los nombres simples como desecho.

En conclusión, las secuencias en las que el adjetivo es temático conservan propiedades eventivas $\mathrm{y}$, por tanto, desde el punto de vista formal, no se lexicalizan. Los sintagmas que contienen un adjetivo clasificatorio, por el contrario, tienen, por la pérdida de propiedades eventivas del núcleo, cierto grado de lexicalización formal. De todos modos, el grado de fijación que pueden alcanzar no es nunca equivalente al de los sintagmas nucleados por un nombre que carece por completo de rasgos formales o semánticos típicos de un verbo ${ }^{3}$.

\footnotetext{
${ }^{2}$ En el caso de los nombres creados a partir de derivación regresiva, en cambio, la pluralización es en general posible y no señala ningún contraste como el descripto (Cf. Kornfeld \& Resnik, 1999). Esta diferencia morfológica explica el contraste entre enfoques, gastos o costos ambientales y *difusiones ambientales e ?interpretaciones ambientales.

${ }_{3}^{3}$ Un nombre puede tener rasgos típicos de un verbo por derivación morfológica (i.e. deverbales) o por propiedades eventivas de su significado (como es el caso de terremoto, catástrofe, conferencia, curso). Así, las posibilidades de lexicalización de gasto Organon, Porto Alegre, $n^{\circ}$ 26, 1998 


\section{Revisión del fenómeno de reducción de sintagmas N+A}

En textos sobre medio ambiente, las secuencias de la forma nombre deverbal-adjetivo relacional aparecen frecuentemente retomadas sólo por el nombre: gasto ambiental $\rightarrow$ gasto, degradación ambiental $\rightarrow$ degradación. Este fenómeno ha sido descripto como reducción, esto es, "la alteración de la secuencia lineal de un sintagma terminológico pleno mediante la elisión de uno o más de sus constituyentes" (ADELSTEIN \& KUGUEL, 1998).

Ahora bien, como hemos observado anteriormente, las secuencias con núcleo deverbal y adjetivo temático no están lexicalizadas formalmente, por lo que no constituyen verdaderos "sintagmas terminológicos". Por ejemplo, la condición necesaria para la interpretación de contaminación es que la información acerca de la entidad afectada por el evento 'contaminar' esté disponible para el receptor. No es necesario que aparezca la secuencia sintáctica particular contaminación hídrica; puede aparecer cualquier secuencia de significado equivalente (contaminación de las aguas, contaminar las aguas) o la mención en el cotexto -o aun la presuposición contextual- de la entidad afectada (agua). En efecto, no hay criterio metodológico que permita determinar, ante la ocurrencia de contaminación, si se trata de una reducción de contaminación hídrica o de contaminación de las aguas.. Es por eso que, en estos casos, no puede hablarse con propiedad de "reducción del sintagma terminológico", a causa de la relación libre entre los constituyentes de la secuencia.

En cambio, las secuencias como difusión ambiental, educación ambiental, costo ambiental, gasto ambiental, concientización ambiental (nombre resultativo-adjetivo clasificatorio), tienen, por la pérdida de propiedades eventivas del núcleo, cierto grado de lexicalización; por lo tanto, cuando alguno de estos sintagmas aparece en un texto retomado sólo por el nombre, se trata verdaderamente de una reducción. Por otra parte, como se ha observado anteriormente, el adjetivo clasificatorio cumple en estos sintagmas la función de ubicar el nombre en un dominio específico, de ahí la presencia reiterada de ambiental en los sintagmas de este tipo. En ciertas situaciones de comunicación, resulta innecesario especificar el dominio y, entonces, el sintagma se reduce al nombre. La reducción, por lo tanto, dependerá de condiciones cotextuales (mención previa del sintagma pleno en el mismo texto) o contextuales (presuposición del dominio involucrado por la misma situación de comunicación) (ADELSTEIN, KUGUEL et al., 1999).

\section{Aplicaciones}

La distinción teórica que hemos establecido tiene algunas consecuencias aplicadas en el tratamiento terminográfico de las secuencias nombre-adjetivo.

Las secuencias con nombre deverbal eventivo-adjetivo temático, como ya se ha visto, no están lexicalizadas formalmente. Su inclusión en un glosario se justifica sólo por la frecuencia con la que coocurren sus componentes, vale decir, por su carácter de colocaciones. Los nombres que los integran tienen, independientemente de las secuencias que nucleen, rasgos de significado especializado (contaminación, erosión), aun cuando provengan del léxico común (protección, vigilancia). Por lo tanto, son los nombres, y no las secuencias, los que deben aparecer como entradas. Dentro del cuerpo del artículo, figurarán las secuencias $\mathrm{N}+\mathrm{A}$ o $\mathrm{N}+\mathrm{prep}+\mathrm{N}$ de mayor frecuencia de aparición en textos especializados. En los casos en que a partir del nombre se generen paradigmas de secuencias (por ejemplo, contaminación ambiental/ contaminación del ambiente, contaminación hídrical contaminación del agua/ contaminación de las aguas, contaminación sonora, etc.), estas se tratarán como subentradas en la medida en que se asocie a ellas algún rasgo conceptual específico (por ejemplo, el límite a partir del cual se considera que la entidad está afectada por la contaminación).

Los sintagmas con nombre deverbal resultativo-adjetivo clasificatorio, en cambio, deben ser registrados como entradas en su forma plena. Ello se debe, por un lado, a que la pérdida de propiedades eventivas en el núcleo hace que haya cierto grado de lexicalización formal. Por otro lado, el nombre por sí mismo no tiene rasgos de significado especializado (por ejemplo, gasto, difusión, enfoque, costo), de modo que la pertenencia al dominio de conocimiento específico es señalada únicamente por el adjetivo.

ambiental (que tiene rasgos verbales por derivación morfológica) son semejantes a las de delito ambiental o riesgo ambiental (que tienen rasgos verbales por su significado eventivo).

Organon, Porto Alegre, nº 26, 1998 


\section{Recapitulación}

En este trabajo, hemos intentado presentar una caracterización de dos tipos de secuencia nombre-adjetivo a partir de propiedades formales y semánticas. La distinción establecida entre colocaciones y sintagmas con cierto grado de lexicalización es el resultado de la interpretación de estas propiedades a través de la noción de eventividad. En el nivel teórico, este trabajo pretende ser un aporte a la discusión más general acerca de las propiedades formales y semánticas asociadas con las categorías gramaticales básicas: nombre y verbo. En el nivel aplicado, a partir de la distinción establecida, revisamos el fenómeno de la reducción para proponer pautas para el reconocimiento y el tratamiento de formas alternativas de designación de un mismo concepto.

\section{Anexo: Clasificación de secuencias N+A (a partir del adjetivo)}

Adjetivos no derivados

\begin{tabular}{|l}
\hline Lluvia ácida \\
marea negra \\
marea roja \\
presupuestos mínimos \\
tóxicos agudos \\
residuos sólidos \\
impacto positivo \\
impacto negativo \\
\hline
\end{tabular}

Adjetivos derivados

\section{I. DENOMINALES}

2.I.A. relacionales

2.I.A.a temáticos

asentamientos humanos

cambio climático

contaminación ambiental

contaminación hídrica

control ambiental

daño ambiental

deterioro ambiental

diagnóstico ambiental

emanación tóxica

erosión eólica

erosión hídrica

impacto ambiental

ordenamiento territorial

participación pública

presión demográfica

protección ambiental

vigilancia ambiental

\section{I.A.b. clasificatorios}

actores sociales

agricultura urbana

agua superficial

ambiente biológico

ambiente físico

ambiente socio cultural

animadores sociales

\section{II. DEVERBALES \\ 2.II.A. pasivos \\ área protegida \\ especies amenazadas \\ oxígeno consumido \\ oxígeno disuelto}

\section{II.B. activos}

actividad productiva

agentes cancerígenos

agente mutagénico

cuerpo receptor

especie indicadora

ganadería transhumante

ganadería extensiva

ganadería intensiva

bosque nativo

energías alternativas

material radiactivo

residuo radiactivo

\section{II.C. con 'ble'}

área intangible

cosecha sostenible

desarrollo sustentable

manejo sustentable

población mínima viable

recursos no renovables

recursos renovables 


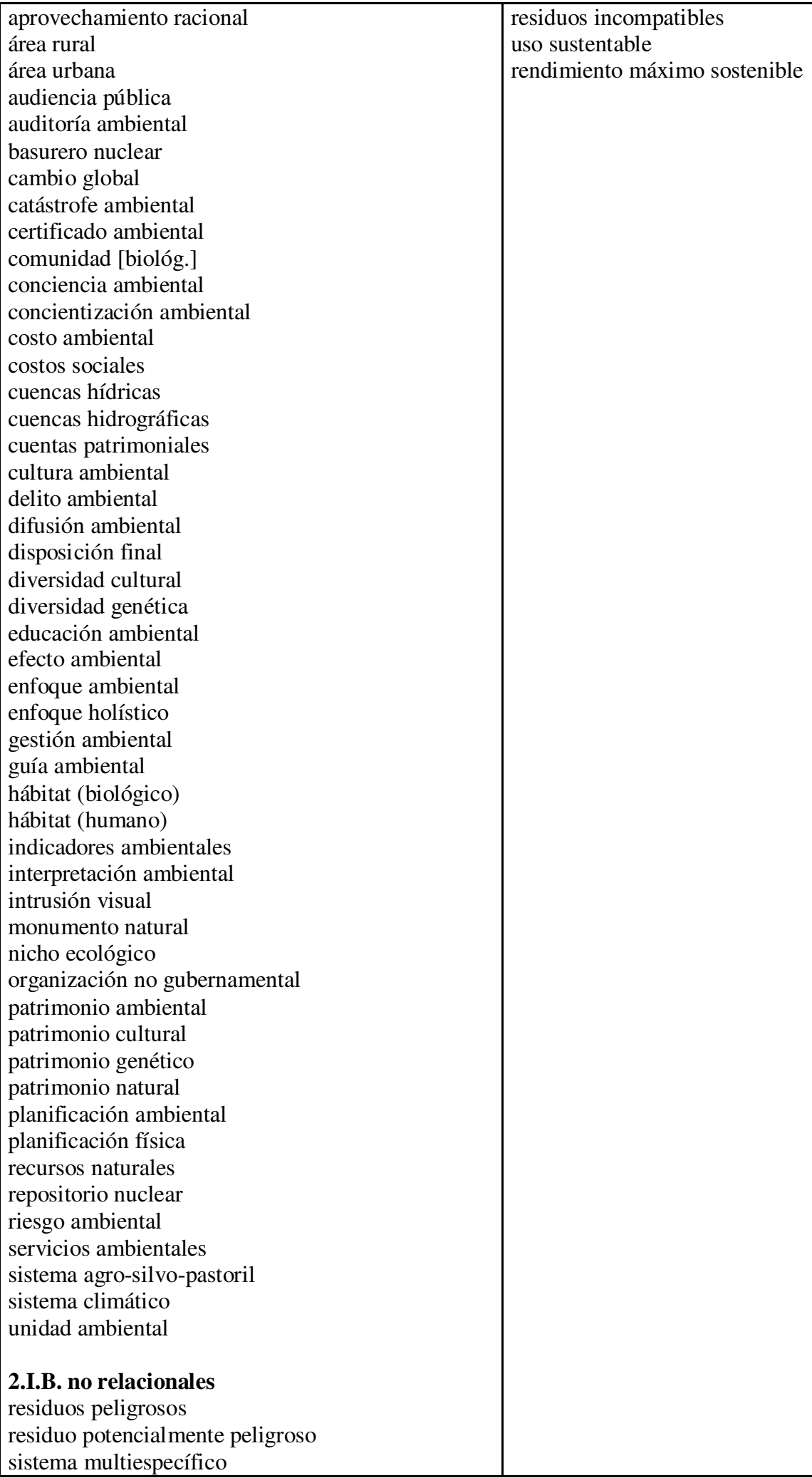

2.I.B. no relacionales

residuos peligrosos

residuo potencialmente peligroso

sistema multiespecífico

residuos incompatibles

uso sustentable

rendimiento máximo sostenible 


\section{BIBLIOGRAFÍA}

ADELSTEIN, A. (1998) “Condiciones de reductibilidad léxica de los sintagmas terminológicos", VI Simposio Iberoamericano de Terminología, 16 al 20 de noviembre de 1998, Universidad de La Habana (Cuba) (en prensa).

ADELSTEIN, A. e I. Kuguel (1998) "La reducción de sintagmas terminológicos”, Actas del Seminario Terminología y Mercosur. Recursos léxicos para la terminología. Buenos Aires: Secretaría de Ciencia y Tecnología de la Nación-Universidad Nacional de General Sarmiento, págs. 42 a 56.

ADELSTEIN, A., I. Kuguel, L. Kornfeld y G. Resnik (1999) "El sintagma terminológico: propiedades formales y semánticas”, Terceras Jornadas de Terminología y Traducción, Universidad Nacional

de

Tucumán, 20 al 22 de septiembre de 1999 (en prensa).

ALONSO, H., N. Bricchetto de Lara y R. Palota (coords.) (1995) Glosario Ambiental. Buenos Aires: Secretaría de Recursos Naturales y Ambiente Humano de la Nación (Sistema de consulta de Glosario Ambiental Versión 0).

BOSQUE, I. y C. Picallo (1996) "Postnominal adjectives in Spanish DPs", Journal of Linguistics 32, Cambridge University Press. 349-385.

DI SCIULLO, A. M. y E. Williams (1987) On the Definition of Word. Cambridge: MIT Press.

KORNFELD, L. y G. Resnik (1998) "Los sintagmas nominales con preposición en la terminología de medio ambiente”, Actas del Seminario Terminología y Mercosur. Recursos léxicos para la terminología. Buenos Aires: Secretaría de Ciencia y Tecnología de la Nación-Universidad Nacional de General Sarmiento, págs. 26 a 41.

KORNFELD, L. y G. Resnik (1999) "Límites entre sintaxis y morfología: los sintagmas nominales con preposición”, Congreso Internacional La Gramática. Modelos. Enseñanza. Historia, 4 al 6 de agosto de 1999 (en prensa).

KUGUEL, I. (1998) "La reducción léxica de sintagmas terminológicos en el contexto discursivo", VI Simposio Iberoamericano de Terminología, 16 al 20 de noviembre de 1998, Universidad de La Habana (Cuba) (en prensa). 